\title{
Use of recreational Viagra among men having sex with men
}

\author{
R Crosby, R J DiClemente
}

Sex Transm Infect 2004;80:466-468. doi: 10.1136/sti.2004.010496

\begin{abstract}
Objective: Given the potential for Viagra (sildenafil) use to foster greater friction during sex (owing to enlarged erection size) and prolonged sex, the recreational use of this substance warrants investigation in the context of STI risk. Thus, an exploratory study was conducted to identify bivariate correlates of recreational (non-prescription) Viagra use among men who have sex with men (MSM) attending a popular sex resort for men located in the southern United States.

Methods: A cross sectional study was conducted. Behavioural measures, including Viagra use, were assessed using a 3 month recall period.

Results: Of 164 men asked to participate, 91\% completed a self administered questionnaire. Men resided in 14 states, most of which were located in the southern United States. Their average age was 40 years. Most (93\%) men self identified as white. The median annual income interval was $\$ 25000$ to $\$ 50000$. One sixth (16.7\%) reported being HIV positive. 16\% reported using non-prescription Viagra. Age $(p=0.41)$, income $(p=0.32)$, and HIV serostatus $(p=0.85)$ were not associated with Viagra use. Of men recently using ecstasy during sex, $35 \%$ reported Viagra use compared to $13 \%$ among those not using ecstasy $(p=0.01)$. Of men recently using cocaine during sex, $37 \%$ reported Viagra use compared to $13 \%$ among those not using cocaine $(p=0.009)$. Use of "poppers" approached, but did not achieve, statistical significance as a correlate of Viagra use $(p=0.06)$. Recent frequency of unprotected anal sex $(p=0.79)$, fisting $(p=0.10)$, rimming $(p=0.64)$, and having five or more sex partners $(p=0.09)$ were not associated with Viagra use.

Conclusion: Recreational Viagra use was relatively common among men, regardless of age or HIV serostatus. Viagra use was associated with men's substance abuse behaviours rather than their sexual risk behaviours.
\end{abstract}

M en who have sex with men (MSM) experience a disproportionate risk of infection with HIV. ${ }^{1-4}$ Evidence also suggests that MSM are disproportionately at risk of acquiring and transmitting bacterial STIs. ${ }^{5-10}$ Addressing these threats to MSM involves multiple challenges, many are behavioural. For example, it is quite plausible that recreational use of Viagra (sildenafil) among MSM may be an important contributing factor to their risk of STI acquisition. Studies in the United Kingdom and the United States indicate that recreational Viagra use has become common among MSM. ${ }^{11-15}$ Further, it has been suggested that Viagra should be sold with warnings relative to increased risk of STI. ${ }^{16}$ The suggestion may have a great deal of merit as use of Viagra for anal insertive intercourse among MSM may lead to two conditions that could conceivably foster tissue abrasion and therefore increase the odds of STI transmission: greater friction during sex (from enlarged erection size) and prolonged sex.

From a behavioural perspective, it is important to identify correlates of recreational Viagra use among MSM. Empirical investigations of this question have been uncommon. However, a recent study (in San Francisco, CA, USA) found that Viagra use among MSM was associated with illicit drug use, being HIV positive, older age, and having unprotected sex with risky partners. ${ }^{15}$ Given the need to expand this body of research, we conducted an exploratory study designed to identify bivariate correlates of recreational (non-prescription) Viagra use among MSM attending a sex resort located in the southern United States.

\section{METHODS}

\section{Study sample}

During Saturdays from May through November of 2002, 164 men attending a sex resort located in northeast Georgia were randomly approached by trained male research staff and asked to participate in a brief survey. Of these, 150 completed a self administered questionnaire (yielding a response rate of $91 \%)$. Incentives for participation were not provided. The Emory University institutional review board approved the study protocol before study implementation. A more detailed explanation of the sample can be found in two articles that have addressed distinctly different research questions using these data. ${ }^{17}{ }^{18}$

\section{Data collection}

Men giving informed consent were provided with a 10 page (single sided and double spaced) questionnaire. Men completed the questionnaire at tables within the patio area or near the pool.

\section{Measurement of recreational Viagra use}

Using a 3 month recall period, men were asked if they had "used non-prescription Viagra for sexual encounters."

\section{Measurement of correlates}

In addition to assessing self reported HIV status and two demographic correlates (age and income level), we assessed correlates related to substance abuse behaviour and sexual behaviour. All recall periods were limited to the past 3 months. Substance abuse behaviour consisted of three questions asking men about their use of "poppers" (alkyl nitrites, including isobutyl nitrite, butyl nitrite, and amyl nitrite that cause muscles around blood vessels to relax), ecstasy (methylenedioxymethamphetamine), and cocaine. Assessed sexual practices were rimming, engaging in group sex, fisting, having sex with five or more partners during the recall period, and engaging in unprotected anal sex.

Abbreviations: MSM, men who have sex with men; STI, sexually transmitted infections 


\section{Data analysis}

Associations between dichotomous correlates and Viagra use were assessed by contingency table analyses. Associations between correlates measured on a continuous level and Viagra use were assessed by independent groups $t$ tests. Significance was defined by an alpha level of 0.05 or less.

\section{RESULTS}

\section{Characteristics of the sample}

Men residing in 14 states comprised the sample (most states were within a 500 mile radius of the sex resort). Average age of the men was 40.6 years (SD 9.4; median 40 years; range $45)$. Most (92\%) men self identified as white. The median income annual interval was $\$ 25000$ to $\$ 50000$ (the equivalent of $€ 20875$ to $€ 41759$ ). One sixth (16.7\%) reported they were HIV positive. Men reported having sex with an average of 10 partners in the past 3 months (SD 42; median 4; range 500). Seven men did not answer the question about Viagra use; thus, valid data for the analysis were obtained from 143 men. Twenty three men (16.1\% of 143) reported using non-prescription Viagra in the past 3 months.

\section{Bivariate associations}

Table 1 displays the percentage of men reporting nonprescription Viagra use stratified by their responses (yes versus no) to the assessed dichotomous correlates. Table 1 also provides prevalence ratios, their $95 \%$ confidence intervals, and respective $\mathrm{p}$ values. As shown, two indicators of substance abuse achieved significance (ecstasy and cocaine), with use of poppers approaching significance $(p=0.06)$. With the exception of fisting $(p=0.10)$ and engaging in sex with five or more partners $(p=0.09)$, none of the other correlates approached significance.

Differences in age between men who reported nonprescription Viagra use (mean age 39.1) and men not reporting use (mean age 40.9) were not observed $(t=0.83$; degrees of freedom $=140 ; p=0.41)$. Similarly, differences in income levels were not observed $(t=1.0$; degrees of freedom $=136 ; p=0.32$ ).

Because statistical power was limited, an effect size analysis for the correlates approaching significance was warranted. Effect size, as assessed by Cohen's h, has been described as a useful method for comparing proportions. ${ }^{19} 20$ Cohen has described an $\mathrm{h}$ value of 0.20 as representing small effects and noted that medium effects begin at approximately 0.50 . Fisting produced an $\mathrm{h}$ value of 0.35 and the correlate pertaining to five or more sex partners yielded an $h$ value of 0.30 . These effects are modest at best. Similarly, the effect for poppers was modest $(h=0.31)$. By comparison, the two statistically significant effects yielded a medium effect size ( $h=0.53$ for ecstasy and $h=0.57$ for cocaine)

\section{DISCUSSION}

This exploratory study of MSM found that recreational Viagra use was associated with substance abuse (ecstasy and cocaine) rather than age, income, HIV status, or selected sexual risk behaviours. In particular, the findings suggest that MSM who attend sex resorts may use or not use recreational Viagra regardless of their sexual risk behaviours. However, the findings also suggest that MSM who obtain and use other illicit substances (for example, ecstasy and cocaine) may be especially likely to also obtain and use Viagra on a recreational basis. Thus, in STI prevention education efforts, recreational Viagra use may be a particularly important issue to address for MSM who abuse substances.

Other than the observed associations with substance abuse, our findings were markedly different from the findings reported from a sample of MSM in San Francisco. ${ }^{15}$ While the differences may be attributable to the different culture of MSM in the southern United States compared to San Francisco, a more likely explanation is the unique nature of our sample: men attending a sex resort.

\begin{tabular}{|c|c|c|c|c|c|}
\hline Correlate & No & $\begin{array}{l}\% \text { Reporting } \\
\text { Viagra use }\end{array}$ & $\mathbf{P R}^{*}$ & $95 \% \mathrm{Cl}+$ & p Value \\
\hline \multicolumn{6}{|c|}{ Knowingly HIV positive } \\
\hline Yes & 23 & 17.4 & & & \\
\hline No & 120 & 15.8 & 1.09 & $0.41-2.93$ & 0.85 \\
\hline \multicolumn{6}{|c|}{ Used poppersł } \\
\hline Yes & 71 & 21.1 & & & \\
\hline No & 71 & 9.9 & 2.14 & $0.93-4.94$ & 0.06 \\
\hline \multicolumn{6}{|c|}{ Used ecstasy $\ddagger$} \\
\hline Yes & 20 & 35.0 & & & \\
\hline No & 119 & 12.6 & 2.78 & $1.30-5.95$ & 0.01 \\
\hline \multicolumn{6}{|c|}{ Used cocaine } \\
\hline Yes & 19 & 36.8 & & & \\
\hline No & 122 & 13.1 & 2.81 & $1.33-5.92$ & 0.009 \\
\hline \multicolumn{6}{|c|}{ Engaged in rimming $\ddagger$} \\
\hline Yes & 87 & 26.4 & & & \\
\hline No & 56 & 14.3 & 1.84 & $0.85-3.99$ & 0.64 \\
\hline \multicolumn{6}{|c|}{ Engaged in group sex $\ddagger$} \\
\hline Yes & 90 & 16.7 & & & \\
\hline No & 52 & 15.4 & 1.08 & $0.49-2.38$ & 0.84 \\
\hline \multicolumn{6}{|c|}{ Engaged in fisting $\ddagger$} \\
\hline Yes & 22 & 27.3 & & & \\
\hline No & 120 & 13.1 & 2.04 & $0.90-4.65$ & 0.10 \\
\hline \multicolumn{6}{|c|}{ Five or more sex partners $\ddagger$} \\
\hline Yes & 69 & 21.7 & & & \\
\hline No & 72 & 11.1 & 1.96 & $0.89-4.32$ & 0.09 \\
\hline \multicolumn{6}{|c|}{ Engaged in unprotected anal sex $\ddagger$} \\
\hline Yes & 68 & 17.6 & & & \\
\hline No & 72 & 12.5 & 1.41 & $0.63-3.14$ & \\
\hline
\end{tabular}

*Prevalence ratio. †Confidence interval. $\ddagger$ Past 3 months. 
Regardless, further empirical investigations addressing recreational Viagra use among MSM are warranted. Further research should test prevention messages and programmes that emphasise to MSM the potential for Viagra to increase their risk of acquiring or transmitting STIs, including HIV. A priority population for these studies may be MSM who abuse other substances including alcohol and methamphetamines (especially given the potential for fatal drug interactions in conjunction with non-prescription Viagra use). Men may, for example, benefit from messages that simply explain how prolonged and chemically exaggerated vasocongestion of the penis can foster microabrasions that increase risk of STI acquisition and transmission.

\section{Limitations}

Findings are limited by several factors, including the inherent limitations of a cross sectional study design and the use of a convenience sample. An important limitation is reliance on the validity of men's responses to the interview questions. Further, the analysis was limited by the low statistical power. However, our assessment of effect size indicated that the study had adequate power to detect medium or larger effect sizes.

\section{Conclusions}

About one of every six men reported recent use of nonprescription Viagra. This recreational use was associated with men's substance abuse behaviours. Specifically, MSM who use ecstasy and cocaine may be more likely to also use Viagra on a recreational basis. Given the potential for Viagra to increase risk of STI, prevention education efforts may be warranted.

\author{
Authors' affiliations \\ R Crosby, College of Public Health, Division of Health Behavior, \\ University of Kentucky, KY, USA \\ R J DiClemente, Rollins School of Public Health, Department of \\ Behavioral Sciences and Health Education, GA, USA \\ R J DiClemente, Emory Center for AIDS Research, GA, USA \\ R J DiClemente, Emory University School of Medicine, Department of \\ Pathology and Laboratory Medicine, GA, USA
}

Correspondence to: Richard Crosby, PhD, College of Public Health, 121 Washington Avenue, Room 111C, Lexington, KY 40506-0003, USA; crosby@uky.edu

Accepted for publication 6 May 2004

\section{REFERENCES}

1 Centers for Disease Control and Prevention. Need for sustained HIV prevention among men who have sex with men. Available online at www.cdc.gov/hiv/pubs/facts/msm.htm. Accessed on 19 November 2001.

2 Centers for Disease Control and Prevention. HIV incidence among young men who have sex with men-seven US cities, 1994-2000. Morb Mort Wkly Rep 2001;49:440-3.

3 Centers for Disease Control and Prevention. HIV/AIDS among racial/ethnic minority men who have sex with men-United States, 1989-1998. Morb Mort Wkly Rep 2000;49:4-11.

4 Sullivan PS, Chu SY, Fleming PL, et al. Changes in AIDS incidence for men who have sex with men, United States 1990-1995. AIDS 1997;11:1641-6.

5 Centers for Disease Control and Prevention. Sexually transmitted disease surveillance, 2000. Atlanta, GA: US Department of Health and Human Services, 2001.

6 Rietmeijer CA, Judson FN, Douglas JM, et al. Increasing rates of gonorrhea (GC) and other sexually transmitted infections (STI) among men who have sex with men (MSM) compared to men who have sex with women (MSW) in the Denver Metro Health Clinic (DMHC): a 10-year trend analysis. Int J STD AIDS $2001 ; 12: 77$

7 Centers for Disease Control and Prevention. Gonorrhea among men who have sex with men-- selected sexually transmitted disease clinics, 1993-1996. Morb Mortal Wkly Rep 1997;46:889-92.

8 Fox KK, del Rio C, Holmes KK, et al. Gonorrhea in the HIV era: A reversal of trends among men who have sex with men. Am J Public Health 2001;91:959-64.

9 Centers for Disease Control and Prevention. Resurgent bacterial sexually transmitted disease among men who have sex with men-King County, Washington, 1997-1999. Morb Mortal Wkly Rep 1999;48:773-7.

10 Centers for Disease Control and Prevention. Outbreak of syphilis among men who have sex with men-Southern California, 2000. Morb Mortal Wkly Rep 2001;50:117-20.

11 Sherr L, Bolding G, Maguire M, et al. Viagra use and sexual risk behaviour among gay men in London. AIDS 2000;14:2051-3.

12 Aldridge J, Measham F. Sildenafil (Viagra) is used as a recreational drug in England. BMJ 1999;318:669.

13 Mansergh G, Colfax GN, Marks G, et al. The circuit party men's health survey: findings and implications for gay and bisexual men. Am J Public Health 2001;91:953-8.

14 Colfax GN, Mansergh G, Guzman R, et al. Drug use and sexual risk behavior among gay and bisexual men who attend circuit parties: a venues based comparison. J AIDS 1999;28:373-9.

15 Chu PL, McFarland W, Gibson S, et al. Viagra use in a community-recruited sample of men who have sex with men, San Francisco. J AIDS 2003;33:191-3.

16 Anonymous. San Francisco asks FDA to place STD warning on Viagra. AIDS Policy Law 2002;17:8.

17 Crosby RA, DiClemente RJ, Mettey A. Correlates of recent unprotected anal sex among men having sex with men attending a large sex resort in the south. Sex Transm Dis 2003;30:909-13.

18 Mettey A, Crosby RA, DiClemente RJ, et al. Associations between internet sexseeking and STI-associated risk behaviors among men who have sex with men. Sex Transm Infect 2003;79:466-8.

19 Cohen J. Statistical power analysis for the behavioral sciences, 2nd ed. Hillsdale, NJ: Lawrence Erlbaum Associates, 1988.

20 Crosby RA, Rothenberg R. In STI interventions, size matters. Sex Transm Infect 2004;80:80-2. 